\title{
Housing condition and seizure susceptibility in Mongolian gerbils
}

\author{
HARRIETT KAPLAN \\ New York State Institute for Basic Research in Developmental Disabilities, Staten Island, New York
}

In a study on the effect of housing condition on seizures in the IBR/SP colony of inbred seizure-prone Mongolian gerbils, pups housed alone at the early age of 3 weeks exhibited more frequent and more severe seizures than their paired counterparts. Gerbils housed in complex environments from birth to 4 weeks of age underwent fewer seizures than gerbils continuously housed in standard cages. Gerbils housed in standard cages from birth to 10 weeks of age and then housed in complex environments showed a marked decrease in seizure frequency after the change. While their previous frequency $(\mathbf{7 3 \%})$ was similar to that of controls $(88 \%)$, their subsequent frequency $(21 \%)$ was significantly lower than that of controls $(80 \%)$ and seizures were less severe. These results indicate that a very powerful, nonpharmacological inhibitor of gerbil seizures is continuous housing in an environment that provides daily opportunity for greater stimulation and/or activity than is normally provided laboratory animals.

Seizure-prone Mongolian gerbils undergo epileptiform seizures when subjected to stressful situations (Kaplan, 1975; Thiessen, Lindzey, \& Friend, 1968). Experimental manipulations such as handling and extra stimulation, which have been shown to modify an animal's response to stress (Denenberg, 1964; Levine, 1962), also tend to reduce the frequency and severity of seizures (Kaplan \& Miezejeski, 1972; Kaplan \& Silverman, 1978). The way animals are housed, for example, housing density and/or complexity, has been shown to affect stress-related activities, including seizures. For example, Berg, Shanin, and Hull (1975) found that gerbil pups that were weaned at 15 days of age and isolated in cages that provided minimal sensory cues spent "more time in seizures and/or freezing behavior" than did pups housed with nonlactating foster parents and sibs. However, it was not clear whether the results were due to early weaning, reduction of sensory stimulation, lack of opportunity for social interactions, or a combination of these variables. Pettijohn (1978) housed adult gerbils alone, in pairs, or in groups of four. After 12 weeks of differential housing, there was a significant difference in seizure susceptibility among the three groups. Gerbils housed alone underwent seizures during $55 \%$ of their tests, paired gerbils underwent them during $34 \%$ of their tests, and grouped gerbils, during $28 \%$ of their tests. This was

\footnotetext{
Thanks are extended to Greg Panarello and Patricia Yak for testing the animals, George Psaltis for help in feeding and maintaining our colonies, Richard Weed and Lucille Donadio for drawing and photographing the figures, and Lucille Corso for typing the manuscript. The author's mailing address is: N.Y.S. Institute for Basic Research in Developmental Disabilities, 1050 Forest Hill Road, Staten Island, New York 10314.
}

in contrast to a mean of $\mathbf{2 5 \%}$ for each group before differential housing.

The following experiments were undertaken to investigate further the effects of housing condition on seizure susceptibility in seizure-prone gerbils. Experiment 1 compared the effects of individual vs. paired housing imposed early in development. Experiments 2 and 3 studied the effects of housing gerbils in an environment more complex than a standard laboratory cage. In Experiment 2, the experimental gerbils were born and housed in a complex environment until 4 weeks of age, at which time they were weaned and housed in standard laboratory cages. In Experiment 3 , the experimental gerbils were born and housed in standard cages until 10 weeks of age, when they were paired and housed in the complex environment.

\section{EXPERIMENT 1}

\begin{abstract}
Method
Subjects were 85 Mongolian gerbils (Meriones unguiculatus) from 25 litters from the N.Y.S. Institute for Basic Research's seizure-prone colony (IBR/SP gerbils). Development and maintenance of this colony has been described earlier (Kaplan, 1981). Littermates were represented in at least two of the four following conditions: weaned at 4 weeks and housed alone in a small 28.5 $\times 17.8 \times 12.7 \mathrm{~cm}$ cage $(15$ males +8 females); weaned at 4 weeks and paired with a gerbil of the same sex in a large $48 \times 27 \times$ $15 \mathrm{~cm}$ cage (18 males +9 females-a nonexperimental female was paired with the odd female); weaned at 3 weeks and housed alone in a small cage ( 11 males +6 females); weaned at 3 weeks and paired in a large cage ( 12 males +6 females). Starting at 6 weeks of age, each subject was given a standard seizure test concurrent with cage cleaning every other week for a total of 15 tests. For the test, the subject was placed into a $48 \times 38 \times 20 \mathrm{~cm}$. clear-plastic open box for $3 \mathrm{~min}$ and then into a clean home cage in which the first 3 min were considered as part of the 6-min standard seizure test. For paired subjects, the clean home cage was separated into two
\end{abstract}


chambers by a clear plastic divider for this part of the seizure test. The following measures were recorded: latency to seizure onset, duration of seizure, and seizure severity based on a standardized checklist with ratings of 0.5 to 5.0 , depending on the number of specific motor behaviors observed (Kaplan, 1981).

The data were analyzed using 2 (weaning age) $\times 2$ (housing condition) analyses of variances and follow-up t tests.

\section{Results and Discussion}

There were no significant main effects, but a significant interaction between weaning age and housing condition $[\mathrm{F}(1,81)=5.13, \mathrm{p}<.05]$ was found. Gerbils weaned at 3 weeks and housed alone had a mean $( \pm S D)$ seizure frequency of $11.5( \pm 2.4)$, which was significantly higher than the mean frequency $9.3( \pm 2.8)$ of gerbils weaned at 3 weeks and paired $[t(33)=2.45, p<.02]$. In contrast, there was no difference between gerbils weaned at 4 weeks and housed alone (mean frequency $9.8 \pm 3.0$ ) or housed in pairs (mean frequency $10.5 \pm 2.9$ ). Figures $1 \mathrm{a}$ and $1 \mathrm{~b}$ illustrate this interaction. A similar significant interaction was found with respect to seizure severity $[F(1,81)=5.41, p<.05]$. In this instance, gerbils weaned at 3 weeks and housed alone had a mean
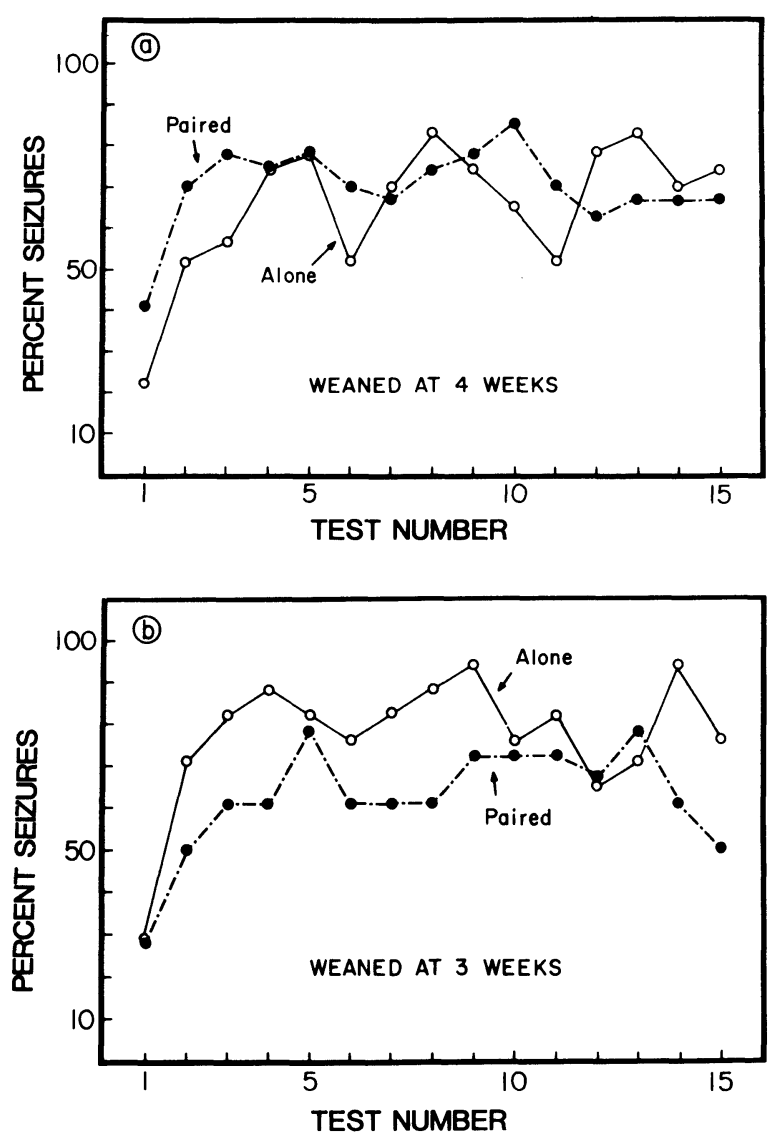

Figure 1. Percentage of subjects undergoing selzures during each of the 15 standard selzure tests: (a) subjects weaned at 4 weeks and housed alone or in pairs, and (b) subjects weaned at 3 weeks and housed alone or in pairs. seizure severity score of $3.4( \pm 0.40)$, which was significantly greater than the mean severity score of 2.9 $( \pm 0.41)$ of gerbils weaned at 3 weeks and paired $[t(33)=4.05, p<.01]$. Gerbils weaned at 4 weeks had a mean score of $3.1( \pm 0.49)$ if housed alone and of $3.1( \pm 0.44)$ if paired.

The group weaned early and housed alone differed from the other groups in two other respects. First, it was the only group in which there were fatalities. Three died before testing began and one soon after. Second, we found that 6 of the 17 survivors were highly reactive, and difficult to catch and handle throughout the experiment. Only one gerbil in the early-paired group exhibited similar behavior. Using Fishers exact test, we determined that this difference was significant $(p=.0360)$. Of the groups weaned at 4 weeks, none of the paired gerbils and 6 of the 23 individually housed gerbils were noted to be difficult to handle during the first two tests. Subsequently, only one animal persisted in this behavior. There was no difference among the groups in any other measure, and nor were there differences between males and females in this or in the following two experiments.

These results indicate that it is only when IBR/SP gerbils are housed alone at an early age that they exhibit more frequent and more severe seizures than gerbils that are paired. By the time the gerbil is 4 weeks of age, it has changed to the extent that depriving it of a cagemate no longer has the same effect it had earlier. However, having a greater number of cagemates at this age does appear to have an effect.

In an earlier study (Kaplan, 1979), gerbils weaned at 4 weeks of age and housed in groups of four until 10 weeks of age and then paired, underwent seizures in $90 \%$ of their tests. This was significantly higher than that of gerbils weaned at 4 weeks and housed individually. Seizure susceptibility in this latter group was $68 \%$, which is similar to that of both individually housed gerbils $(65 \%)$ and paired gerbils $(70 \%)$ in the present experiment, which were also weaned at 4 weeks. This suggests that at different ages there may be differences in the optimal amount of social stimulation required for modulation of seizure susceptibility.

\section{EXPERIMENT 2}

\section{Method}

The subjects were 34 IBR/SP gerbils. The 20 experimental (E) gerbils ( 12 males +8 females) were born and housed until weaning in a complex environment (the habitat). This was a two-story plastic and wire-mesh structure equipped with a coiled wire tube that provided access between the stories (Figure 2). The 14 control (C) subjects ( 7 males and 7 females) were born and continuously housed in standard plastic cages. All $E$ and $C$ subjects were weaned at 4 weeks and housed in same-sex pairs in standard cages. From 6 weeks of age on, all subjects were tested for occurrence of seizures every other week for 30 weeks (15 tests), using the standard seizure test described in Experiment 1. Maintenance of animals and the measures recorded were the same as those in Experiment 1. 


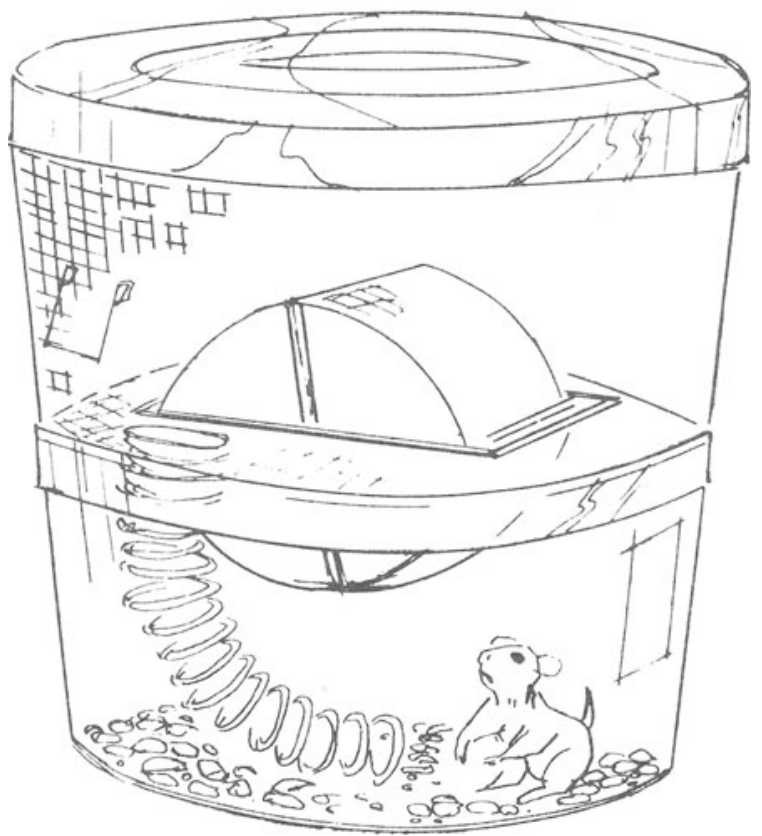

Figure 2. The complex environment (habitat) in which $\mathbf{E}$ (Experiment 2) and $\mathbf{H}$ (Experiment 3) gerbils were housed. These were purchased from the General Cage Corp. (Elwood, Indiana) as Varmit Cities, Model 301, but are no longer available. Corn-cob bedding and paper towels were provided in the lower level.

\section{Results and Discussion}

E gerbils (housed in the habitat from birth until 4 weeks of age) underwent seizures during $60 \%$ of their tests, whereas $\mathbf{C}$ gerbils (housed continuously in standard cages) underwent seizures during $75 \%$ of their tests $[\mathrm{t}(32)=2.48, \mathrm{p}<.02]$. Other measures, such as latency to seizure and seizure severity, were not significantly different.

Thus, gerbils housed in a complex environment from birth until weaning underwent significantly fewer seizures than did gerbils housed in standard cages during this period. The fact that the difference was small and that there was no concomittant effect on seizure severity may possibly be attributed to two factors. One is that the pups utilized the entire environment only during their last week in it, the time their eyes opened (at about 20 days of age) and they became more active and mobile than previously. The second is that some of the effects of living in a complex environment have been shown to be reversible (Rosenzweig \& Bennett, 1978). This is an important consideration, since the $\mathrm{E}$ animals were no longer in the experimental environment during the period they were being tested.

Experiment 3 examined the effects of housing animals in a complex environment (habitat) at a later age, for a longer period of time, and testing them during the same period in which they were housed in the experimental environment.

\section{EXPERIMENT 3}

\section{Method}

The subjects were 32 IBR/SP gerbils, 16 males and 16 females. These animals were born and reared in standard $48 \times 27 \times 15 \mathrm{~cm}$ plastic cages. After weaning at 4 weeks, they continued to be housed in these cages in pairs of the same sex. At 6, 8, and 10 weeks of age, each subject was given a standard seizure test (described in Experiment 1). At 10 weeks of age, littermates were mated. Eight of these pairs (Group S) were housed in standard cages, and the remaining 8 pairs (Group $\mathrm{H}$ ) were housed in habitats. Testing for seizures was continued every other week for an additional 12 tests. Maintenance of animals and the measure recorded are described in Experiment 1 . Student $t$ tests were used for between-groups comparisons and tests for correlated samples were used for within-group comparisons.

\section{Results and Discussion}

Figure 3 illustrates the percentages of Group $\mathrm{H}$ and Group $S$ animals having seizures during their biweekly tests. There were no significant differences in seizure activity between Group $\mathrm{H}$ and Group $\mathrm{S}$ during their first three seizure tests when all the animals were housed in standard cages. The mean number of seizures in Group $\mathrm{H}$ during these tests was $\mathbf{2 . 2}$ $( \pm 0.75)$, or $73 \%$; in Group $S$, it was $2.6( \pm 0.50)$, or $88 \%$. After Group H gerbils were housed in the habitat, there was a gradual decline in seizure susceptibility during the next three tests and then a precipitous decline, which was maintained during the remainder of the experiment (Figure 3). The mean number of seizures in Group $\mathrm{H}$ gerbils during these 12 tests was $2.6( \pm 2.3)$, or $21 \%$, which was significantly different from their prehabitat performance $[t(15)$ for correlated means $=6.1, p<.01]$. This was also significantly different from the mean number of seizures, $9.6( \pm 2.1)$, or $80 \%$, exhibited by gerbils in Group $S$ during their last 12 tests $[\mathrm{t}(30)=9.0, \mathrm{p}<$ $.01]$. In contrast to Group $H$, seizure activity in Group S gerbils during their first 3 tests was not sig-

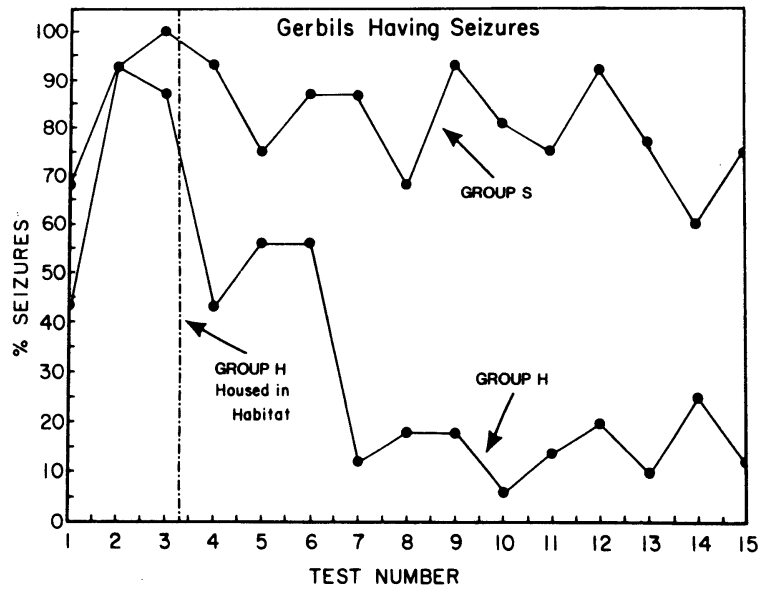

Figure 3. Percentage of seizures during each of the 15 standard seizure tests in $\mathbf{H}$ (habitat-housed) and $\mathbf{S}$ (standard-caged) subjects before and after $\mathrm{H}$ subjects were put into the habitat. 
nificantly different from that during their subsequent 12 tests.

Group S animals did, however, exhibit a significant increase in seizure severity, from a mean rating of $2.3( \pm 0.52)$ during the first three tests to a mean rating of $3.4( \pm 0.62)$ during their subsequent 12 tests $[t(15)=6.4, p<.01]$. This is similar to the increase seen in most IBR/SP gerbils during this period under standard laboratory conditions. No such increase was observed in Group $\mathrm{H}$ animals, which had a mean rating of $2.3( \pm 0.27)$ during the first three tests and a mean rating of $2.2( \pm 0.56)$ during the subsequent 12 tests. Mean rating during these 12 tests was significantly different in Groups $H$ and $S[t(27)=5.4$, $\mathrm{p}<.01]$. Group $\mathrm{H}$ gerbils did show a significant increase in seizure latency from a mean of $50 \mathrm{sec}( \pm 53)$ before to a mean of $110 \mathrm{sec}( \pm 74)$ after habitat housing $[t(12)=2.45, p<.05]$. (Since three $H$ gerbils did not have seizures after being housed in the habitat, only those animals with scores before and after were used for the above analyses.) No significant increase was seen in Group S animals, which had a mean latency of $58 \mathrm{sec}( \pm 43)$ during their first three tests and a mean latency of $69 \mathrm{sec}( \pm 34)$ subsequently.

We have found that 10-week-old IBR/SP gerbils, reared and housed in the same manner as the rest of our IBR/SP colony and with a similar susceptibility to undergo seizures, will show a gradual but ultimately dramatic decrease in seizure susceptibility after they are housed in a more complex environment. In these animals, seizure frequency dropped more than $50 \%$, latency to seizure increased, and seizure severity which normally increases during this period, remained the same.

\section{GENERAL DISCUSSION}

In all three experiments, there tended to be an inverse relationship between complexity of the environment and seizure susceptibility. Housing complexity has been shown to affect such variables as endocrine function and brain anatomy, weight, and chemistry (e.g., Green, Greenough, \& Schlumph, 1983; Levine, 1962; Rosenzweig \& Bennett, 1978; Walsh, 1980) as well as behavior and seizures (e.g., Brain \& Benton, 1979; Schreiber, 1971). Bennett, Diamond, Krech, and Rosenzweig (summarized in Rosenzweig \& Bennett, 1978) have identified some of the mechanisms by which environmental variables could affect these changes in physiology and behavior. Furthermore, they found that gerbils responded to differential environments in a way very similar to that of rats and mice, on which most of the studies were performed (Rosenzweig \& Bennett, 1969). These effects-increased cortical weight, greater dendritic branching, and increased number of dendritic spines-could decrease seizure susceptibility in several ways. They might, for example, cause a change in the gerbil's response to the stressful stimuli that trigger seizures, they might suppress the activity of epileptogenic neurons, or they might inhibit the spread of abnormal neuronal activity.

These experiments, in conjunction with earlier work from this laboratory and that of others, have provided evidence that an extremely powerful inhibitor of gerbil seizure activity is the opportunity to interact with an environment that is a more complex and therefore more stimulating than the one normally provided laboratory animals. Whether these results have implications for the nonpharmacological treatment of human beings with epilepsy depends on the degree to which the gerbil model will be shown to conform to one or more of the various forms of human epilepsy.

\section{REFERENCES}

Berg, R. A., Shanin, R. D., \& Hull, E. M. Early isolation in the gerbil (Meriones unguiculatus): Behavioral and physiological effects. Physiological Psychology, 1975, 3, 35-38.

Brain, P., \& Benton, D. The interpretation of physiological correlates of differential housing in laboratory rats. Life Sciences, 1979, 24, 99-116.

Denenberg, V. H. Critical periods, stimulus input, and emotional reactivity: A theory of infantile stimulation. Psychological Reviews, 1964, 71, 335-351.

Green, E. J., Greenough, W. T., \& Schlumph, B. E. Effects of complex or isolated environments on cortical dendrites of middle-aged rats. Brain Research, 1983, 264, 233-240.

KAPLAN, H. What triggers seizures in the gerbil, Meriones unguiculatus? Life Sciences, 1975, 17, 693-698.

KAPLAN, H. Postweaning housing condition affects seizure activity in the Mongolian gerbil. Society for Neuroscience $A b$ stracts, 1979, 5, 194.

KaPlan, H. Effects of fostering on seizure activity in the Mongolian gerbil. Developmental Psychobiology, 1981, 14, 565-570.

Kaplan, H., \& Miezejeski, C. Development of seizures in the Mongolian gerbil (Meriones unguiculatus). Journal of Comparative and Physiological Psychology, 1972, 81, 267-273.

Kaplan, H., \& Silverman, W. P. Early experience affects seizure latency and postseizure recovery time in the Mongolian gerbil. Neuropsychologia, 1978, 16, 649-652.

LEvine, S. The effects of infantile experience on adult behavior. In A. J. Bachrach (Ed.), Experimental foundations of clinical Psychology. New York: Basic Books, 1962.

Petrijorn, T. F. Influence of social group size on seizure frequency in Mongolian gerbils. Journal of General Psychology, 1978, 99, 149-150.

Rosenzweig, M. R., \& Bennett, E. L. Effects of differential environments on brain weights and enzyme activities in gerbils, rats, and mice. Developmental Psychobiology, 1969, 2, 87-95.

Rosenzweig, M. R., \& Bennett, E. L. Experimental influences on brain anatomy and brain chemistry in rodents. In G. Gottlieb (Ed.), Studies in the development of behavior and the nervous system (Vol. 4): Early influences. New York: Academic Press, 1978.

Schreiber, R. A. Effect of housing density on the incidence of audiogenic seizures in DPA/2J mice. Journal of Comparative and Physiological Psychology, 1971, 76, 300-304.

Thiessen, D. D., Lindzey, G., \& Friend, H. C. Spontaneous seizures in the Mongolian gerbil (Meriones unguiculatus). Psychonomic Science, 1968, 11, 227-228.

WALSH, R. N. Effects of environmental complexity and deprivation on brain chemistry and physiology: A review. International Journal of Neuroscience, 1980, 11, 77-89.

(Manuscript received June 5, 1983; revision accepted for publication August 1, 1983.) 